\title{
Caregiver Perspectives of Parenting Children Affected by Trauma
}

\author{
Jason C. Lawson, Janeene C. Sibla \\ University of Mary, Bismarck, USA
}

\begin{abstract}
The purpose of this pilot research study was to explore the perspectives of caregivers who parent children affected by trauma, and to identify sensory processing concerns within this population. This study included transcribed interviews of six caregivers, and Sensory Profile Caregiver Questionnaires of 12 children who have experienced various types of traumatic events. Following are the four themes supported by the data collected: (1) Every caregiver voiced the importance of providing structure and routine to their child(ren)'s daily life; (2) Several caregivers voiced sensory aversions that occurred on a daily basis that affected their child(ren)'s ability to complete daily routines; (3) All the caregivers reported concerns with their child(ren)'s ability to interpret and follow social cues; and (4) Every caregiver reported using creative and flexible parenting strategies unique to their family's circumstances. The Sensory Profile results assisted with triangulating the qualitative data obtained from the caregiver interviews. Sensory Profile results identified high percentages of emotional reactivity $(83.3 \%)$ and difficulty with emotional/social responses (75\%). The results of this study indicated that children who experienced trauma display a wide array of challenging and intense behaviors, which impact daily life. Suggestions for future research include a larger sample size with more diversity in age, ethnicity, and socioeconomic background. The addition of a control group would assist in identifying statistically significant differences between children who have and have not experienced trauma. The results of this study may be utilized by caregivers, healthcare professionals, teachers, and others in order to provide effective parenting strategies and interventions for the behavioral and sensory processing issues that may arise when working or caring for children affected by trauma.
\end{abstract}

Keywords: caregivers, parenting, children, trauma, sensory profile, sensory processing

Approximately five million children in the US experience trauma every year (Child Trauma Academy, 2010) and one in four children will witness or experience a traumatic event before they reach 4 years of age (Finkelhor, Turner, Shattuck, \& Hamby, 2013). Subsequently, it is likely that healthcare providers, teachers, and caregivers will encounter these children in their daily work. Therefore, it is imperative that these professionals and caregivers have the skills necessary to understand and manage the unique characteristics presented by children who have witnessed or experienced trauma.

Trauma is referred to as a physical or psychological threat or assault to a child's physical integrity, sense of self, personal safety, or to the safety of another person significant to the child (Da Silva, 2011). Trauma includes various types of abuse, neglect, and disruptions in the household (Middlebrooks \& Audage, 2008).

Jason C. Lawson, Ph.D., MS, OTR/L, Assistant Professor, Department of Occupational Therapy, University of Mary. Janeene C. Sibla, OTD, MS, OTR/L, Professor, Department of Occupational Therapy, University of Mary. 
The most common types of traumatic experiences reported are the loss of loved ones, witnessing domestic violence, and living with a caregiver whose caregiving ability is impaired (The National Child Traumatic Stress Network, 2013).

Forty percent of children who experience trauma encounter repeated or multiple accounts of traumatic experiences (The National Child Traumatic Stress Network, 2013). Children exposed to repeated or multiple accounts of trauma develop behaviors that can affect their daily lives and can persist throughout adulthood (The National Child Traumatic Stress Network, 2013). Common behaviors of children who experience trauma include shutting down or dissociating, demonstrating hypervigilance or perseveration, experiencing exaggerated fight or flight responses, hyperarousal, and demonstrating a disrupted attachment process to caregivers, which may negatively impact the child's physical, cognitive, emotional, and social growth (Cole, Greenwald, Geron, Joel, Luray, \& Michael, 2005; Schore, 2002). Furthermore, children who experience trauma may exhibit defiance, aggression towards others, self-injurious behaviors, impulsive or destructive behaviors, and poor social skills or peer relationships (Cole et al., 2005; Goldfinch, 2009). Several studies also show that experiencing childhood trauma affects overall brain development that decreases the child's ability to process various types of information (U.S. Department of Health and Human Services, Administration for Children and Families, Administration on Children, Youth and Families, Children's Bureau, 2009; Goldfinch, 2009; Schore, 2002). In fact, researchers have begun reporting concerns with sensory processing in children who experienced trauma (Da Silva, 2011; Moore, 2006; Perry, 2000). However, little empirical evidence exists regarding sensory processing concerns in children who have witnessed or experienced trauma.

Sensory processing occurs when the brain integrates all of the body's senses to accurately respond to environmental stimuli. Therefore, when people have difficulty interpreting the sensory information they are receiving, a sensory processing disorder may exist (Bundy, Lane, \& Murray, 2002). Moore (2006) estimated that individuals who experienced trauma had an $80 \%$ chance of developing a sensory processing disorder.

When a child is unable to process sensory information accurately, concerns with gross and fine motor skills, cognitive development, and emotional/social responses are affected, which may further contribute to a child's academic, social and emotional functioning (Ben-Sasson, Carter, \& Briggs-Gowen, 2009). Furthermore, children with sensory processing disorders may demonstrate limited attention, hyperactivity, hypoactivity, seeking or avoiding oral input, issues with fine or gross-motor coordination, and defensiveness or avoidance of textures and/or movement (Koomar \& May-Benson, 2006). These sensory processing behaviors are likely to impact children's ability to function in day to day life, requiring appropriate intervention from professionals and caregivers.

Cermak and Daunhauer (1996) studied sensory processing of children who had experienced neglect in orphanages. The children residing in the orphanages had difficulty self-organizing, self-regulating social and emotional situations, regulating their activity level, and tolerating self-feeding. Researchers concluded that children exposed to insufficient childcare, inadequate shelter, and a minimal diet showed greater concerns with touch processing, avoiding or excessively seeking movement and stimuli, as well as demonstrating visual and auditory problems. Therefore, this study provides rationale for investigating sensory processing among children who have experienced or witnessed various types of trauma.

\section{Parenting a Child Who has Experienced Trauma}

Studies indicate that parenting influences a child's wellbeing (Banyard, England, \& Rozelle, 2001; 
Gewirtz, Forgatch, \& Wieling, 2008). According to Gewirtz et al. (2008), caregivers of children who experienced trauma are often the only available support to their child following the traumatic event. Researchers agree that parenting a child who has experienced trauma requires different parenting strategies compared to parenting children who have not experienced trauma (Alisic, Boeije, Jongmans, \& Kleber, 2012; Hughes, 2006; Purvis, Cross, \& Sunshine, 2007; Wesselmann, Schweitzer, \& Armstrong, 2014). Utilizing parenting styles and strategies that are appropriate for children who experienced trauma has the potential to positively affect the child's recovery and adjustment to the trauma (Gewirtz et al., 2008). Alisic et al. (2012) suggested specific parenting strategies for caregivers of children who have experienced trauma. The parenting strategies include: returning to normal routines after stressful events, giving the child opportunities to talk without pressure, answering questions honestly and at the child's level, and providing enjoyable activities for leisure. Caregivers who use appropriate parenting strategies for children who have experienced trauma allows their children to develop a strong, healthy sense of self (Goldfinch, 2009). Although there are a few studies that provide specific parenting strategies for parenting children affected by trauma, a limited number of studies report the lived experiences of caregivers who are parenting these children. Caregivers of children affected by trauma have the potential to report successful parenting strategies, and to identify further concerns for caring for their children. Acquiring this information could further assist professionals and caregivers identify appropriate caregiving practices and interventions to assist these children in their daily lives.

\section{Purpose of the Study}

The purpose of this pilot study was to explore the lived experience of caregivers who parent children affected by trauma, and to identify the incidence of sensory processing concerns among these children. Caregivers are appropriate individuals to report on their lived experiences of parenting children who have witnessed or experienced trauma as they have the most direct contact with these children in their natural environments.

\section{Methodology}

This pilot study was approved by the Institutional Review Board at a university in the Upper Midwest. The researchers explored the lived experiences of caregivers who parent children affected by trauma by conducting semi-structured interviews with theses caregivers. Researchers also aimed to identify sensory processing concerns among children who have experienced trauma by having caregivers complete the Sensory Profile Caregiver Questionnaire (Dunn, 1999). Informed consent was obtained from the participants prior to their participation in this study.

\section{Participants}

Participants were recruited through a local outpatient pediatric mental health facility. The licensed professional clinical counselor (LPCC) at the outpatient facility informed caregivers of children between the ages of 3 to 10 years who have experienced trauma about the research project and acquired an informed consent for their participation in the study. Twelve caregivers completed the Sensory Profile Caregiver Questionnaire and six caregivers were interviewed.

Caregivers were all Caucasian, and included biological parents, stepparents, foster parents, grandparents, and significant others of biological parents. The children reportedly experienced: physical abuse $(n=2)$, witnessing a traumatic event $(n=3)$, sexual abuse $(n=1)$, physical and sexual abuse $(n=3)$, and a combination 
of neglect, physical abuse, and witnessing a traumatic event $(n=3)$. The gender of the children included six males and six females. The age ranges of children being reported on were 3 to 4 years 11 months $(n=2), 5$ to 7 years 11 months $(n=4)$, and 8 to 10 years 11 months $(n=6)$. Three of the children were Native American, and nine were Caucasian. There were no other medical diagnoses of the children reported by their caregivers.

\section{Procedure}

Quantitative data. Quantitative data were obtained from caregivers of children who experienced trauma by completing the Sensory Profile Caregiver Questionnaire (Dunn, 1999). This questionnaire contains 125 items that describe children's responses to daily sensory experiences. Caregivers completed the form by indicating the frequency of the child's responses ("Always", "Frequently", "Occasionally", "Seldom", or "Never") to various sensory experiences (Dunn, 1999). The caregiver responses were then categorized into Factor and Section Summaries, which were scored as typical performance (84th percentile or greater), probable difference (between 2nd and 16th percentile), and definite difference (below the 2nd percentile). A table was constructed with the frequencies and percentages of participants who scored as "typical performance", "probable difference", and "definite difference" within each Sensory Profile Caregiver Questionnaire Factor and Section Summaries.

Qualitative data. Qualitative data were obtained through individual, semi-structured interviews with caregivers of children who experienced trauma. Interviews were completed in a quiet and confidential location. Interview questions were aimed to explore the caregivers' lived experiences of parenting a child who experienced trauma. Clarifying questions were asked in order to gain further insight into each caregiver's experience. Interviews lasted approximately 30 to 45 minutes, and were recorded and transcribed verbatim for accuracy. Data were divided into meaningful segments, labeled as codes, categories, and then developed into themes.

\section{Results}

\section{Quantitative Results}

Table 1 represents the frequencies of the Sensory Profile Caregiver Questionnaire Factor Summaries scores and percentages of the combined "probable" and "definite" differences scores.

Table 1

Frequencies of Sensory Profile Caregiver Questionnaire Factor Summaries Scores

\begin{tabular}{lccll}
\hline Factor summaries & Typical performance & Probable difference & Definite difference & $\begin{array}{l}\text { Percentage of probable } \\
\text { and definite differences }\end{array}$ \\
\hline Sensory seeking & 4 & 6 & 2 & 66.7 \\
Emotionally reactive & 2 & 6 & 4 & 83.3 \\
Low endurance/tone & 5 & 3 & 4 & 58.3 \\
Oral sensory sensitivity & 11 & 0 & 1 & 8.3 \\
Inattention/distractibility & 4 & 5 & 3 & 66.7 \\
Poor registration & 7 & 4 & $\mathrm{I}$ & 41.7 \\
Sensory sensitivity & 9 & 3 & 0 & 25.0 \\
Sedentary & 12 & 0 & 0 & 0.0 \\
Fine motor/perceptual & 10 & 2 & 0 & 16.7 \\
\hline
\end{tabular}

In four out of nine Factor Summaries, over 50\% of the Sensory Profile Caregiver Questionnaires were scored at a probable or definite difference. The greatest percentage of scores with the probable and definite 
differences were with "Emotionally Reactive" (83.3\%), "Sensory Seeking" (66.7\%), and
"Inattention/Distractibility" (66.7\%).

Table 2 represents the frequencies of the Sensory Profile Caregiver Questionnaire Section Summaries scores and percentages of the combined "probable" and "definite" differences scores.

Table 2

Frequencies of Sensory Profile Caregiver Questionnaire Section Summaries Scores

\begin{tabular}{lllll}
\hline $\begin{array}{l}\text { Section Summaries } \\
n=12\end{array}$ & $\begin{array}{l}\text { Typical } \\
\text { performance }\end{array}$ & $\begin{array}{l}\text { Probable } \\
\text { difference }\end{array}$ & $\begin{array}{l}\text { Definite } \\
\text { difference }\end{array}$ & $\begin{array}{l}\text { Percentage of } \\
\text { probable and definite } \\
\text { differences }\end{array}$ \\
\hline Auditory processing & 6 & 4 & 2 & 50.0 \\
Visual processing & 9 & 2 & 1 & 25.0 \\
Vestibular processing & 5 & 3 & 4 & 58.3 \\
Touch processing & 6 & 5 & 1 & 50.0 \\
Multisensory processing & 6 & 4 & 2 & 50.0 \\
Oral sensory processing & 9 & 2 & 1 & 25.0 \\
Sensory processing related to endurance/tone & 5 & 3 & 4 & 58.3 \\
Modulation related to body position and movement & 6 & 4 & 2 & 50.0 \\
Modulation of movement affecting activity level & 8 & 4 & 0 & 33.3 \\
Modulation of sensory input affecting emotional responses & 6 & 3 & 3 & 50.0 \\
Modulation of visual input affecting emotional responses and & 7 & 4 & 1 & 41.7 \\
activity level & 3 & 6 & 3 & 75.0 \\
Emotional/social responses & 4 & 5 & 3 & 66.7 \\
Behavioral outcomes of sensory processing & 9 & 2 & 1 & 25.0 \\
Items indicating thresholds for response & & & 2 & 2 \\
\hline
\end{tabular}

In nine out of 14 Section summaries, greater than or equal to $50 \%$ of the Sensory Profile Caregiver Questionnaires were scored at a probable or definite difference. The greatest percentage of scores with the probable and definite differences were with "Emotional/social responses" (75\%) and "Behavioral outcomes of sensory processing" $(66.7 \%)$.

\section{Qualitative Results}

Caregiver interviews were analyzed by creating codes, categories, and themes. Figure 1 illustrates how the code were condensed into categories, and the categories formed relationships that contributed to the four major themes.

The four themes that emerged from the qualitative data analysis are as follows: (1) caregivers identified a significant need for structure in children's daily routines to avoid or prevent challenging behaviors; (2) Behaviors related to the child's sensory aversions negatively affected the child's ability to participate in daily activities; (3) Decreased social skills of their children affected peer interactions; and (4) utilizing flexible parenting strategies enabled caregivers to adapt to the challenging needs of their children.

Structure and routine. All participants identified having a specific morning, evening, and bedtime routine for their children. Caregivers reported that a predictable routine assisted their children to be successful with their daily activities. One caregiver described limiting noise and distractions prior to bedtime in order to aid in the transition. The caregiver (of a 10 year old child) stated, "He does quiet time, which is actually lying down, and he falls asleep almost every time. If he doesn't (engage in quiet time) he is like a 2 year old". Another caregiver reinforced the importance of following a routine by stating, “... once we hit a certain point in 
the night, it's always the same routine". Routines are particularly important for children who have experienced trauma because they need environments that are predictable and safe (Cole et al., 2005).

\begin{tabular}{|c|c|c|}
\hline \begin{tabular}{|l} 
Trial \& error \\
Structure \\
Routine \\
Quiet time \\
Communication \\
Patience \\
Avoiding conflict
\end{tabular} & Routine & $\begin{array}{l}\text { Every caregiver voiced the importance of } \\
\text { providing structure and routine to their } \\
\text { child(ren)'s daily life in order to prevent } \\
\text { challenging behaviors. }\end{array}$ \\
\hline \begin{tabular}{|l} 
Behaviors \\
Morning \\
Bedtime \\
Routine \\
Dressing \\
Clothing \\
Textures \\
Perseverates \\
Sensory \\
\end{tabular} & Sensory processing & $\begin{array}{l}\text { Several caregivers voiced sensory } \\
\text { aversions that occurred on a daily basis } \\
\text { that affected their child(ren)'s ability to } \\
\text { complete daily routines. }\end{array}$ \\
\hline \begin{tabular}{|l|} 
Behaviors \\
Social cues \\
Misinterpret \\
Intimidating \\
Left out \\
Conflict \\
Aggressive \\
Roughhouse \\
Immature
\end{tabular} & Social skills & $\begin{array}{l}\text { Every caregiver reported concerns with } \\
\text { their child(ren)'s ability to interpret and } \\
\text { follow social cues, which affected social } \\
\text { interactions. }\end{array}$ \\
\hline \begin{tabular}{|l|} 
Redirection \\
Conflict \\
Opposition \\
Aggression \\
Preparing self \\
Positive reinforcement \\
Supervision \\
Patience \\
Understanding \\
Flexibility \\
Creative
\end{tabular} & Parenting strategies & $\begin{array}{l}\text { Every caregiver reported utilizing } \\
\text { creative and flexible parenting strategies } \\
\text { unique to their family's circumstances in } \\
\text { order to reduce challenging and } \\
\text { unpredictable behaviors. }\end{array}$ \\
\hline
\end{tabular}

Figure 1. Data analysis.

The interviewed caregivers also reported an increase in challenging behaviors during transitions between activities or to new environments. One caregiver described the change his/her child faced when transitioning from a daycare setting, to a school setting. "It was an adjustment for him to go from an in-home daycare to school, which is a real structured environment. He doesn't do good with change at all... he doesn't do well... he gets anxious". According to Cole et al. (2005), children who have experienced trauma display difficulty during times of transition, which may include changing activities, moving to a new home, changing schools, or focusing on the unknown future. During these times of transition, children perceive the environment as out of control, unsafe, and unfamiliar (Benckendorf, 2012).

Sensory aversions. During the interviews, several caregivers identified that their children had strong sensory aversions to textures and styles of clothing that affected their child's ability to engage in daily activities that were part of their daily routines. Once caregiver reported how challenging it was to find the exact texture of clothing his/her child would wear. "We had him try everything on. It's too soft, it's not soft enough, it's not 
this, it's not that". One caregiver explained how his/her child dislikes wearing underwear, and the process they endure to get him to wear them.

"We have like about a 20 minute stretching period for the underwear. They are always too tight or he has to have certain ones. He doesn't like it when they're washed because then they shrink back up, and then we have to stretch them all over again".

Another caregiver reported that his/her child would only wear one pair of shorts; Therefore, he/she attempted to purchase the same pair of shorts for him to wear at night.

"I bought him the exact same pair of shorts for him to wear at nighttime, but apparently there is a slight difference where the drawstring hangs out (the waist band) and he'll just look at that and know it is not the right pair".

Another caregiver voiced frustration with their child's sensory preferences during their morning routine resulting in challenging behaviors. "Yesterday morning, (he) didn't want to get dressed... he can get very defiant and we can end up with lengthy timeouts or it can be a big struggle". Koomar and May-Benson (2006) identified that an aversion or preference to limited textures, styles, or fits of clothing are symptoms of sensory processing disorders. Furthermore, literature supports that individuals who have experienced trauma may avoid various types of sensory stimuli, such as clothing textures and styles (Cermak \& Daunhauer, 1996; Stewart \& White, 2008).

Social skills. Caregivers most commonly reported challenges from dealing with their child's decreased social skills. One caregiver described how his/her child prefers rough play, but cannot identify when play has become too aggressive for the other children, which impacts his peer interactions. "If someone starts getting rough, then, of course, he joins in. And he doesn't understand what's too rough. Then he gets a reputation (for being too rough) and nobody wants to include him". Another caregiver explained how his/her child gets ignored by new children because his behaviors often intimidate those who do not know or understand him. "It's tougher when he's meeting new kids because they don't get why he does what he does". Another caregiver explained that his/her child's emotions interfere with socializing with other children. "All she does is cry. Just trying to rationalize with her, she starts to cry. Plus, she cries all the time whenever she doesn't get exactly what she is wanting". Children may not want to socially engage with children who have experienced trauma secondary to differences in behavior and lack of appropriate communication skills (Cole et al., 2005). Furthermore, Benckendorf (2012) identified that children who have experienced trauma have difficulty tolerating stress or peer conflict, which may contribute to defiant behaviors, anxiety, and aggression during social interactions.

Flexibility in parenting strategies. All caregivers reported using various strategies with their children throughout the day to reduce the frequency of challenging and unpredictable behaviors.

Each caregiver identified strategies that were uniquely effective for their family. One caregiver explained how he/she decided to homeschool her child affected by trauma.

He just can't handle attending school... we have tried. His behaviors get so severe at home following school... We had to call the police several times on him. The school said he was doing fine, but he couldn't hold it together once he got home.

This caregiver also reported how he/she is attempting to facilitate independence with the child during his academic lessons in order to decrease challenging behaviors. 
He is supposed to be in third grade, but we have to take it (school lessons) down into first, kindergarten, or second grade work so it is easier for him. When the lessons are easier, he doesn't need me to sit right next to him as I am trying to get him to figure out what to do on the page. Because in a classroom setting, he doesn't have anyone sitting right next to him answering every question he has. You have to think for yourself some. He is not there yet.

Another caregiver explained how his/her child affected by trauma would affect the other children in the family's attempt to get ready in the mornings. He/she explained how they developed a creative routine as a solution to their child's challenging behaviors in order to allow their other children the ability to get ready for the day.

My husband came up with this brilliant idea of having our child go to work with him in the morning... so he and my husband walk to work. He will do 20 minutes of daily reading and other activities (at husband's work) until I can help the other kids get ready and dropped off for school... Otherwise he would sabotage his other siblings getting ready for school or throw big temper tantrums when it is time to leave so we couldn't get everyone to school on time... This way, he gets a little exercise, one-on-one time with dad... Then I have a 15 minute drive time by myself to kind of prepare myself before I pick him up and drop him off.

Another caregiver explained how his/her child needed some extra time to wake up in the morning, which helped prevent argumentative behaviors. "We'll let him warm up, let him just wake up a little bit (because)... I don't want to fight with him right away in the morning... We have to pick our battles, and find that happy medium". Benckendorf (2012) discussed the importance of caregivers remaining calm in potentially emotionally charged situations in order to prevent evoking memories of the child's past trauma. The interviewed caregivers all reported unique parenting strategies and routines that assisted them in remaining calm and avoiding conflicts with their children who have experienced trauma. By utilizing unique strategies, specific for each of their children, the interviewed caregivers were utilizing components of responsive parenting, which Alisic et al. (2010) described as the ability to identify the child's unique needs and provide solutions to any potential concerns.

\section{Discussion}

Data from the Sensory Profile Caregiver Questionnaire assisted with triangulating data from the caregiver interviews. Overall, all data supported that caregivers experience a variety of challenging behaviors from their children who are affected by trauma. Caregivers reported the need to manage meltdowns, tantrums, opposition, excessive crying, and required assistance from police to aid in dealing with aggressive outbursts.

Data from the Sensory Profile identified the highest percentages of children displayed emotional reactivity, or extreme emotional responses. This data also displayed a high percentage of children experiencing difficulty with social and emotional responses, which suggests they lack specific coping mechanisms for dealing with emotions (Dunn, 1999). Similarly, caregivers reported their children struggled in social situations as they lacked comprehension of social cues. Furthermore, their impulsive and aggressive behaviors often intimidated other children, which affected their ability to build peer relationships and engage in social interactions (Cole et al., 2005; Goldfinch, 2009; Perry, 2000; Schore, 2002).

Caregivers also reported sensory aversions to particular textures or styles of clothing, which impacted daily routines. According to several studies, children who have experienced trauma may avoid textures or styles of clothing, which may be related to a sensory processing disorder (Cermak \& Daunhauer, 1996; Koomar \& May-Benson, 2006; Stewart \& White, 2008). Although, according to the Sensory Profile data, only a small percent of reported children demonstrated sensory sensitivity, or viewing light touch or textures as abrasive or 
threatening (Dunn, 1999). The caregivers adamantly reported that sticking to and following a similar daily routine helped decrease disruptive behaviors. Therefore, one could conclude the texture aversions reported by the caregivers may be related to new or unfamiliar experiences in the child's routine as opposed to an actual sensitivity to the texture itself. Several studies report the importance of following a predictable routine for children who have experienced trauma, as something new or unfamiliar in their routine may illicit a fight or flight response secondary to their traumatic history (Benckendorf, 2013; Perry, 2000; Wesselmann et al., 2014).

A large percentage of children were reported to be sensory seeking, or children who seek out activities and may be perceived as fidgety (Dunn, 1999). Similarly, the data suggested that the children had no concerns with being sedentary, or inactive, which was the only Sensory Profile Factor Summary where all the children scored in the typical performance range. During the interviews, few caregivers reported concerns with their children's activity level that was affecting academic progress. Similarly, some literature has supported that children who have experienced trauma tend to be active or hyper-aroused due to an exaggerated fight or flight response secondary to the neurological changes that occurred within the child as a result of experiencing trauma (Benckendorf, 2013; Perry, 2000; Wesselman et al., 2014).

Caregivers and professionals working with children who have experienced trauma would benefit in gaining knowledge and awareness of the unique challenges and behaviors these children exhibit. Caregivers may also be at risk for secondary trauma, or caregiver burn out from the extenuating demands related to the child's traumatic history (Benckendorf, 2013). Therefore, the results of this study support the need for caregiver education and interventions, including recommendations for effective parenting strategies, developing and following a structured and consistent routine, managing behaviors calmly, and assisting children to learn social cues for increasing successful social interactions.

\section{Limitations}

Although the quantitative and qualitative data were utilized to substantiate findings, a limitation of this study included that it was a pilot study, with a small, and fairly homogeneous sample of caregivers of children who had experienced trauma. This fairly small sample size limits the generalizability and the statistical power of the quantitative data. Furthermore, the facility where participants were obtained were seeking specialized therapy for their children who have experienced trauma; therefore, the behaviors reported may have been more significant than those children who experienced trauma that were not receiving services. The study did not explore the ages of the caregivers, which the ages and prior parenting experiences may have influenced the caregiver perspectives and responses.

\section{Implications for Future Research}

Researchers identified minimal literature regarding parenting children affected by trauma from the caregiver perspective, as well as a lack of empirical evidence surrounding sensory processing concerns among these children. Future research should include a larger sample size with more diversity in age, ethnicity, and socioeconomic background among caregivers of children who experienced trauma, as well as the children themselves. The addition of a control group of children who have not experienced trauma would identify if there are statistically significant differences in sensory processing among children who have and have not experienced trauma. Identifying a statistically significant difference could lead to more specific and all-inclusive treatment options for children affected by trauma. 


\section{Conclusion}

In conclusion, the results of this pilot study indicated that children who experienced trauma display unique and challenging behaviors that can be intense, impulsive, and even aggressive, which can further impact a child's ability to function in day to day life. This study sought to understand the lived experiences of caregivers of children who experienced trauma, and to identify the incidences of sensory processing concerns among these children. The caregivers in this study identified a need for familiar, structured routines and flexibility in their parenting practices to meet the ever changing needs of their children. The results of this study may be utilized by caregivers, healthcare professionals, teachers, and others in order to provide effective parenting strategies and interventions for the behavioral and sensory processing issues that may arise with children affected by trauma.

\section{References}

Alisic, E., Boeije, H. R., Jongmans, M. J., \& Kleber, R. K. (2012). Supporting children after Single incident trauma: Parents' views. Clinical Pediatrics, 51(3), 274-282.

Banyard, V. L., England, D. W., \& Rozelle, D. (2001). Parenting the traumatized child: Attending to the needs of nonoffending caregivers of traumatized. Psychotherapy, 38(1), 74-86.

Benckendorf, K. (2012). Children of trauma: What educators need to know. Retrieved from http://attachmentandintegrationmethods.com/Publications/Children_of_Trauma_2012.pdf

Ben-Sasson, A., Carter, A. S., \& Briggs-Gown, M. J. (2009). Sensory over-responsivity in elementary schools: Prevalence and social-emotional correlates. Journal of Abnormal Child Psychology, 37, 705-716.

Bundy, A. C., Lane, A. J., \& Murray, E. A. (2002). Sensory integration theory and practice. Austin, T.X.: F.A. Davis Company.

Cermak, S. A., \& Daunhauer, L. A. (1996). Sensory processing in the post-institutionalized child. American Journal of Occupational Therapy, 51(7), 500-507.

Child Trauma Academy. (2010). Surviving childhood: An introduction to the impact of trauma. Retrieved from http:childtrauma.org

Cole, S., Greenwald, O. J. M., Geron, G., Joel, R. D., Luray, W., \& Michael, G. (2005). Helping traumatized children learn. Retrieved from http://www.massadvocates.org/documents/HTCL_909.pdf

Da Silva, K. (2011). The sensory treatment approach in dealing with trauma in children: Does itwork? Social Work Theses. Retrieved from http://digitalcommons.providence.edu/socialwrk_students/76/Paper76.

Dunn, W. (1999). The sensory profile user's manual. San Antonio, T.X.: Psychological Corporation.

Gewirtz, A., Forgatch, M., \& Wieling, E. (2008). Parenting practices as potential mechanisms for child adjustment following mass trauma. Journal of Marital and Family Therapy, 34(2), 177-192.

Goldfinch, M. (2009). Putting Humpty together again: Working with parents to help children who have early trauma. Australian and New Zealand Journal of Family Therapy, 30(4), 284-299.

Finkelhor, D., Turner, H. A., Shattuck, A., \& Hamby, S. L. (May 2013). JAMA Pediatrics. Retrieved from http://www.unh.edu/ccrc/pdf/0513\%20PED\%20childhood\%20exposure\%20to\%20violence.pdf

Hughes, D. A. (2006). Building the bonds of attachment: Awakening love in deeply troubled children. New York, N.Y.: Guilford Press.

Koomar, J., \& May-Benson, T. (2006). Educator fact sheet signs and symptoms of sensory processing disorder. Retrieved from http://www.thespiralfoundation.org.

Middlebrooks, J., \& Audage, N. (2008). The effects of childhood stress across the lifespan (CS112953). U.S. Department of Health and Human Services, National Center for Disease Control and Prevention. Retrieved from http://www.cdc.gov

Moore, K. $\quad$ M. (2006). Sensory $\quad$ defensiveness. $\quad$ Retrieved from http://www.sensoryconnectionprogram.com/sensory_defensiveness.pdf

Perry, B. (2000). Traumatized children: How childhood trauma influences brain development. The Journal of the California Alliance for the Mentally Ill, 11(1), 48-51.

Purvis, K. B., Cross, D. R., \& Sunshine, W. L. (2007). The connected child: Bringing hope and healing to your adoptive family. New York, N.Y.: McGraw Hill. 
Schore, A. N. (2002). Dysregulation of the right brain: A fundamental mechanism of traumatic attachment and the psychopathogenesis of posttraumatic stress disorder. Australian and New Zealand Journal of Psychiatry, 36, 9-30.

Stewart, L. P., \& White, P. M. (2008). Sensory filtering phenomenology in PTSD. Depression and Anxiety, 25(1), 38-45.

The National Child Traumatic Stress Network, (2013). What is child traumatic stress? Retrieved from $\mathrm{http} / / / \mathrm{nctsn}$. org/nctsn_assets/pdfs/what_is_child_traumatic_stress.pdf

U.S. Department of Health and Human Services, Administration for Children and Families), Administration on Children, Youth and Families, Children's Bureau. (2009). Child Maltreatment 2010. Retrieved from http://www.acf.hhs.gov/programs/cb/stats_research/index.htm\#can.

Wesselmann, D., Schweitzer, C., Armstrong, S. (2014). Integrative parenting: Strategies for raising children affected by trauma. New York, NY: W. W. Norton \& Company. 